\title{
COVID-19 Presenting with Spontaneous Pneumothorax
}

\author{
Intisar Ahmed ${ }^{1}$, Hunaina Shahab ${ }^{1}$, Muhammad Arslan Ahmed ${ }^{2}$ and Muhammad Sohaib ${ }^{3}$ \\ ${ }^{1}$ Department of Medicine, Section of Cardiology, The Aga Khan University, Karachi, Pakistan \\ ${ }^{2}$ Department of Medicine, The Aga Khan University, Karachi, Pakistan \\ ${ }^{3}$ Department of Anesthesia, The Aga Khan University, Karachi, Pakistan
}

\begin{abstract}
The coronavirus disease 2019 (COVID-19) is caused by severe acute respiratory syndrome coronavirus 2 (SARS-CoV-2). The disease mainly affects respiratory system. Its common clinical findings include fever, cough and shortness of breath. Characteristic radiological features of the disease include peripherally distributed, bilateral ground-glass opacities, predominantly involving the lower lung zones. In this report, we present a case of COVID-19 disease presenting with spontaneous pneumothorax. A 26-year male patient was admitted to the Emergency Department with fever, dry cough, shortness of breath and right-sided chest pain. Radiographic imaging of the patient revealed pneumothorax on the right and peripherally distributed non-homogenous opacification. The patient underwent right lateral tube thoracostomy. COVID-19 was diagnosed on testing of nasopharyngeal swab. In conclusion, spontaneous pneumothorax is one of the rare presentations of COVID-19 pneumonia and should be kept in mind in patients presenting with shortness of breath and chest pain.
\end{abstract}

Key Words: Spontaneous pneumothorax, Corona, pneumonia.

How to cite this article: Ahmed I, Shahab H, Ahmed MA, Sohaib M. COVID-19 Presenting with Spontaneous Pneumothorax. J Coll Physicians Surg Pak 2021; 31(JCPSPCR):CR29-CR31.

\section{INTRODUCTION}

The coronavirus disease 2019 (COVID-19) is caused by severe acute respiratory syndrome coronavirus 2 (SARS-CoV-2). ${ }^{1}$ SARS-CoV-2 is a member of the coronaviridae family and contains a single stranded RNA. ${ }^{1,2}$ In December 2019, a new case of coronavirus infection occurred in Wuhan, China. ${ }^{1}$ The new coronavirus was identified on January 6, 2020 and labelled as 2019-nCoV. ${ }^{3}$ Worldwide, more than $6,057,853$ cases of COVID-19 and 371,166 COVID-19-related deaths have been reported till $1^{\text {st }}$ June $2020 .^{4}$

The clinical features of mild COVID-19 include symptoms common to other viral infections, ${ }^{1}$ while clinical features of severe COVID-19 include pneumonia, acute respiratory distress syndrome (ARDS) and shock. ${ }^{5}$

Chest radiographs show low-density pneumonia, which mostly involves bilateral mid-lower zones. In computed tomography (CT), the most common radiological finding is ground-glass appearance. ${ }^{6}$ In this report, we presenta case of COVID-19 pneumonia, who was admitted with spontaneous pneumothorax and recovered completely.

Correspondence to: Intisar Ahmed, Department of

Medicine, Section of Cardiology, The Aga Khan Univer-

sity, Karachi, Pakistan

E-mail: intisarahmed87@gmail.com

Received: July 12, 2020; Revised: November 29, 2020;

Accepted: December 21, 2020

DOI: https://doi.org/10.29271/jcpsp.2021.JCPSPCR.CR29

\section{CASE REPORT}

A 26-year man was admitted to the Emergency Department (ED) with the complaints of fever, cough, shortness of breath, and right-sided pleuritic chest pain. He was in usual state of health three days back when he started having fever with dry cough. He denied body aches and flu-like symptoms. He took paracetamol for fever. His condition did not get better and he started having right-sided pleuritic chest pain one day before presenting to ED. His past medical history was unremarkable. He did not smoke and there was no travel history in recent past. He was in respiratory distress on presentation. Blood pressure was $110 / 70 \mathrm{mmHg}$, pulse $124 / \mathrm{min}$, respiratory rate $30 / \mathrm{min}$, temperature $38.5^{\circ} \mathrm{C}$, and oxygen saturation $\left(\mathrm{SpO}_{2}\right)$ with finger probe was $88 \%$ on room air. Breathing sounds were decreased on the right side and rest of the examination was unremarkable. Arterial blood gases were done immediately, which revealed a $\mathrm{pH}$ of 7.45 with a partial pressure of carbon dioxide of $22 \mathrm{mmHg}$, partial pressure of oxygen of $66 \mathrm{mmHg}$, and arterial oxygen saturation of $86 \%$ on room air. Onchestradiograph, he was foundtohaveright-sided pneumothorax (Figure 1).

In blood tests, total leucocyte count was $12.06 \times 103 / \mathrm{uL}$, lymphocytes $1.59 \times 103 / \mathrm{uL}$, hemoglobin $15.4 \mathrm{~g} / \mathrm{dL}$, hematocrit $42.4 \%$, platelets $188 \times 103 / \mathrm{uL}$ creatinine $0.91 \mathrm{mg} / \mathrm{dL}$, alanine aminotransferase $13 \mathrm{U} / \mathrm{L}$, aspartate aminotransferase $11 \mathrm{U} / \mathrm{L}$, lactate dehydrogenase $150 \mathrm{U} /$, and procalcitonin was 0.043 $\mathrm{ng} / \mathrm{ml}$. Patient was put on mechanical ventilation in $\mathrm{ED}$ due to hypoxemia and respiratory distress. He underwent right lateral tube thoracostomy for pneumothorax and was connected to thoracic suction. The patient was hospitalised with suspicion of COVID-19 and supportive medical therapy was started. On 
nasopharyngeal swab, SARS-CoV-2 was detected in the polymerase chain reaction (PCR). He was stared on intravenous hydrocortisone and oral hydroxychloroquine. His repeat chest radiograph after 12 hours showed complete resolution of rightsided pneumothorax with expansion of right lung and rightsided peripherally located non-homogenous opacification (Figure 2).

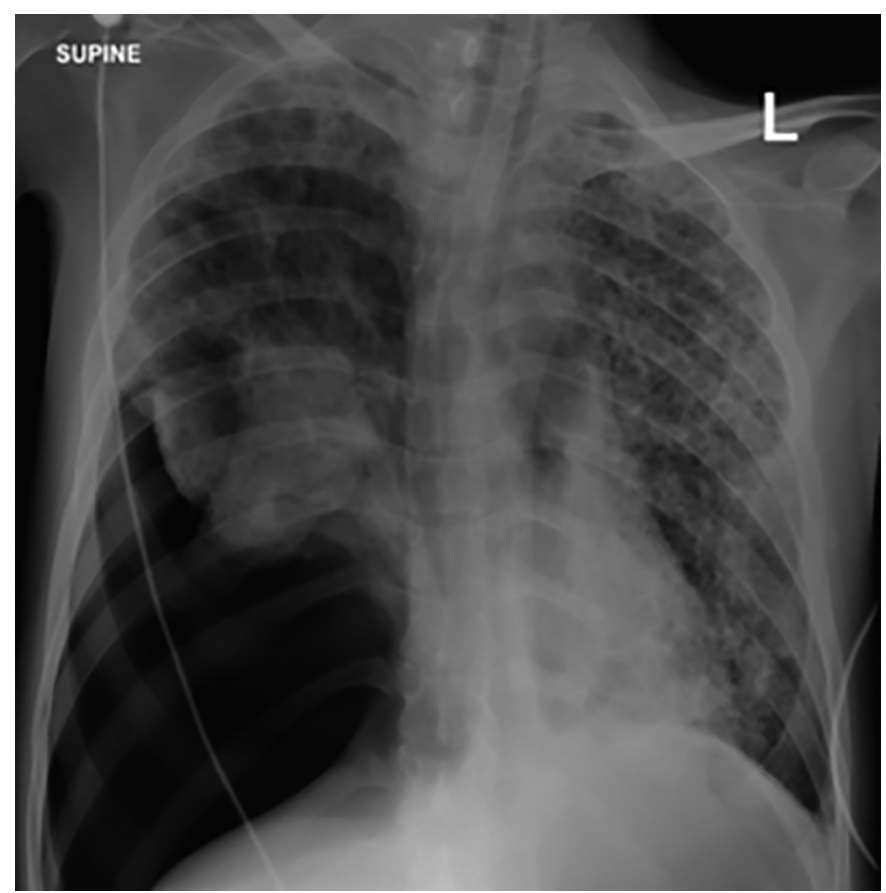

Figure 1: Chest radiograph on presentation showing right-sided pneumothorax with collapsed right lung.

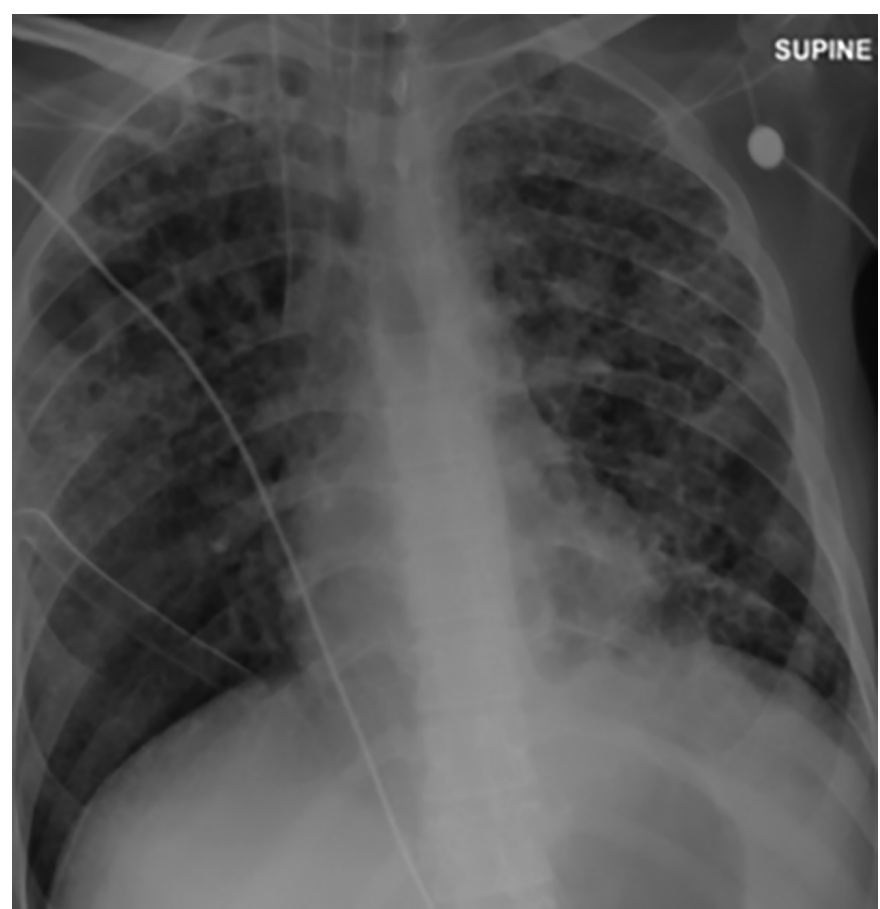

Figure 2: Chest radiograph after right lateral thoracostomy showing resolution of pneumothorax with right-sided opacification.
On second day, leak persisted but patient gradually got better and on third day, was extubated successfully. Other treatment was continued. On chest radiograph, right lung was fully expanded but right-sided opacification continued. Thoracic suction was stopped and he was connected to an underwater seal bottle. Patient was shifted out of intensive care unit and kept in a negative pressure room. On fifth day, he was found to have no leak and right lung was completely expanded. Thoracostomy tube was clamped for six hours and repeatchest radiograph showed no pneumothorax, so tube was removed. Patient was discharged in stable condition on eighth day and advised to remain in home isolation for another week. His repeat nasopharyngeal swab after one week of discharge did not detect SARC-CoV-2. His right lung remained expanded on oneweek follow-up.

\section{DISCUSSION}

Clinical presentations of COVID-19 have ranged from asymptomatic or mild symptoms to severe illness, respiratory distress syndrome and mortality. Common presenting symptoms include fever, cough, and shortness of breath. Other symptoms, such as malaise and respiratory distress, have also been reported. Huang et al. reported fever as the most common symptom followed by cough, myalgia and fatigue. ${ }^{1}$ Headache, sputum production, and diarrhea were reported as rare clinical presentations of this disease. All patients with COVID-19 pneumonia have abnormal lung imaging; and ground-glass opacities are the most common radiographic findings, especially on computed tomography (CT) scan. Pneumothorax has been reported as a very rare presentation of COVID-19.

Aydin et al. reported spontaneous pneumothorax in a young patient with COVID-19. ${ }^{7}$ There were bilateral irregular consolidation areas. There was no underlying lung disease that would have predisposed the patient to pneumothorax. ${ }^{7}$ Salehi et al. investigated imaging findings in 919 COVID-19 positive patients and detected ground-glass densities in $88 \%$ of cases. None of them developed pneumothorax. ${ }^{8}$

In severe acute respiratory failure syndrome, sudden alveolar pressure increase may cause interstitial emphysema and air leak, leading to the development of mediastinal emphysema. ${ }^{9}$ In a descriptive study, Chen et al. describeda case ofCOVID-19 infection, presenting with spontaneous pneumothorax. ${ }^{10}$

In this study, we presented a 26-year man with no underlying structural lung disease. He presented with COVID-19 pneumonia and pneumothorax. Pneumothorax may develop in COVID-19 pneumonia due to alveolar damage. This can cause increased mortality and morbidity. During this pandemic, every patient with dyspnea and fever should bescreened for COVID-19.

\section{PATIENT'S CONSENT:}

A written informed consent has been obtained from the patient to publish data related to this case.

\section{CONFLICT OF INTEREST:}

Authors declared no conflict of interest. 


\section{AUTHORS' CONTRIBUTION:}

All authors have substantially contributed to the conception design, analysis, interpretation of data and drafting of the work. All authors are accountable for all aspects of the work in ensuring that questions related to the accuracy or integrity of any part of the work are appropriately investigated and resolved.

\section{REFERENCES}

1. Huang C, Wang Y, Li X, Ren L, Zhao J, Hu Y, et al. Clinical features of patients infected with 2019 novel coronavirus in Wuhan, China. Lancet 2020; 395(10223):497-506. doi: 10.1016/S0140-6736(20)30183-5.

2. Su S, Wong G, Shi W, Liu J, Zhou J, Liu W, et al. Epidemiology, Genetic Recombination, and Pathogenesis of Coronaviruses. Trends Microbiol 2016; 24:490-502.

3. Li Q, Guan X, Wu P, Wang X, Zhou L, Tong Y, et al. Early transmission dynamics in Wuhan, China, of novel coronavirus infected pneumonia. N Engl J Med 2020; 382(13):1199-1207. doi: 10.1056/NEJMoa2001316.

4. World health organization coronavirus disease 2019 (COVID-19) situation report-133.
5. Wang D, Hu B, Hu C, Zhu F, Liu X, Zhang J, et al. Clinical characteristics of 138 hospitalised patients with 2019 novel coronavirus-infected pneumonia in Wuhan, China. JAMA 2020; 323(11):1061-9. doi: 10.1001/jama.2020.1585.

6. Zheng Ye, Yun Zhang, Yi Wang, Huang Z, Song B. Chest CT manifestations of new coronavirus disease 2019 (COVID19): A pictorial review. Euro Radiol 2020; 30(8):4381-9. doi: 10.1007/s00330-020-06801-

7. Aydın S, Öz G, Humanly A, Balcı A, Gender A. A case of spontaneous pneumothorax in Covid-19 pneumonia. J Surg Resear 2020; 3(2):096-101. DOI: 10.26502/jsr.10020060.

8. Salehi S, Abedi A, Balakrishnan S, Gholamrezanezhad A. Coronavirus disease 2019 (COVID-19): A systematic review of imaging findings in 919 patients. Am J Roentgenol 2020; 215(1):87- 93. doi: 10.2214/AJR.20.23034.

9. Ooi GC, Khong PL, Müller NL, Yiu WC, Zhou LJ, Ho JC, et al. Severe acute respiratory syndrome: temporal lung changes at thin-section CT in 30 patients. Radiology 2004; 230(3):836-44. doi: 10.1148/radiol.2303030853.

10. Chen N, Zhou M, Dong X, Qu J, Gong F, Han Y, et al. Epidemiological and clinical characteristics of 99 cases of 2019 novel coronavirus pneumonia in Wuhan, China: A descriptive study. Lancet 2020; 395(10223):507-13. doi: 10.1016/S0140-6736(20)30211-7. 